\title{
A Suggestion on the Action Mechanisms of Acupuncture Treatment for Controlling Chemotherapy-Induced Peripheral Neuropathy
}

\author{
Hyun-sik Seo, Chang-gue Son, Nam-hun Lee, Jung-hyo Cho* \\ Department of Liver \& Immunology center, College of Korean medicine, Daejeon University
}

\begin{abstract}
Objectives: The purpose of this study is to investigate the mechanism of acupuncture for treating chemotherapy -induced peripheral neuropathy.

Methods: Based on domestic and international papers reported until October 2020, experimental papers on "chemotherapy induced peripheral neuropathy", "mechanism", and "acupuncture" were set up to identify the mechanisms of chemotherapy induced peripheral neuropathy. A total of seven papers were selected and searched: one pilot paper for people and six experimental papers for rats.

Results: In the pilot paper studied by Bao, T., the effect of EA was demonstrated but no significant results were produced for the mechanism. Moon et al. derived the association between EA and plasma $\beta$-endorphin in rat experimental studies on oxalilatin-induced cold hypersensitivity. Meng et al. found relevance to $\mu, \delta$, and $\delta$ opioid through EA stimulation in paclitaxel-induced peripheral neuropathy. Lee et al. studied the relationship between EA and muscarin and 5-HT in rat experiments on oxaliplatin-induced coldness, associated with 5-HT and EA, especially with 5-HT3 receptors. Choi et al. revealed the association of adrenaline and opioid acting on a2- and $\beta$ adrenaline receptors with EA in rat experiments on paclitaxel-induced neuralgia. In rat experiments on oxaliplatin-induced neuralgia reported by Lee, $\beta$-endorphin and encephalin were studied to be mediated by EA. Zhang, T. et al. revealed in the paclitaxel induced rat experiment that EA activates 5-HT.

Conclusion: It is inferred that peripheral neuropathy caused by anticancer drugs can be reduced by activating the action of 5-HT, $\beta$-endorphin, and encephalin through the descending inhibitory pathways. cell differentiation, herbal medicine, Pongamia, stem cells
\end{abstract}

$\overline{\text { Key Words }}$ : Hemotherapy, Peripheral neuropathy, Acupuncture, Mechanism, Descending pathways

\section{Introduction}

As chemotherapy-induced peripheral neuropathy (CIPN) is a serious side effect without a proven standardized treatment option, ${ }^{1)}$ because of the severe symptoms, is the decision to reduce chemotherapy dosage for patients is frequently made. Also the symptoms degrade the quality of life of patients by limiting their mobility and ability to perform everyday life actions. In particular, sensory, motor, and autonomic neurotoxicity could be very disabling or painful. Besides, chronic neuropathy persists in a number of patients for years after chemotherapy cessation. $^{2)}$

To prevent and treat the symptoms of CIPN,

\footnotetext{
- Received : 9 November 2020

- Accepted : 17 November 2020

- Correspondence to: Jung-hyo Cho

East \& West Cancer Medical Center, Korean Medicine Hospital of Daejeon University,

Daejeon, Republic of Korea

Tel : +82-10-3177-5187, Fax : +82-42-477-9007, E-mail :choajoa@dju.kr
} 
calcium and magnesium as well as other pharmacological treatments like gabapentin, antiepileptic, and tricyclic antidepressants have been generally used. However, the symptoms of CIPN such as numbness or paresthesia are not decreased effectively. There is no established prevention and treatment for neuro-regeneration. ${ }^{3)}$

As a result, cancer patients seek additional help for treating their disease or chemotherapy -related side effects. Integrative oncology states that symptoms induced by cancer and cancer treatment could be treated by using non-invasive therapies which are adjuncts to mainstream therapy. The Society of Integrative Oncology Guidelines has set up the range of alternative and complementary therapies including acupuncture, touch therapy, and physical activity. ${ }^{4)}$ These therapies have become plenteous resources in the care of cancer because of their high usage prevalence during active cancer treatments.

In some cases of centers in Europe, forty-seven out of ninety-nine centers provided integrative oncology treatments. In particular, acupuncture has been used in 26 centers $(55.3 \%)$ for cancer -diagnosed patients. ${ }^{5)}$ Acupuncture has been shown to be effective for chemotherapy-induced side effects such as vomiting, fatigue, insomnia, and depression. ${ }^{5)}$ Moreover, acupuncture has been encouragingly used to treat peripheral neuropathy caused by diseases like HIV and diabetes. ${ }^{6}$ )

Likewise, the effectiveness of acupuncture treatment has been admitted still. However, the results of the study are still insufficient and the mechanism of acupuncture treatment to cure CIPN has not been known. Therefore, in this study, we would like to investigate the mechanism of acupuncture treatment on CIPN and discover how acupuncture treatment acts for treating CIPN. Therefore, this study will form the basis for the clinical feasibility of acupuncture treatment in the future.

\section{Methods}

\section{Search Method}

Among the electronic search, we have used websites providing domestic and foreign original text. For researching papers, Google scholar, PubMed, Medline, KISS, and OASIS were used. The targets have been subject to the entire publication in Korean and English dated until October 2020. It only includes research in which the use of electroacupuncture(EA) and acupuncture is contained. Other treatments such as pharmacopuncutre and physical stimulus are excluded.

For researching the mechanisms of acupuncture for chemotherapy induced peripheral neuropathy, the keywords searched were "mechanism" AND "acupuncture" AND "chemotherapy-induced peripheral neuropathy" OR "CIPN". In addition, we also studied from the reference lists of relevant articles for the mechanism of acupuncture for chemotherapy-induced peripheral neuropathy.

\section{Data Collection}

A total of 5,704 articles were detected by electronic searches and the examination of reference lists of the articles for mechanisms of CIPN. We chose the studies from databases through screening the titles and/or abstracts. After screening, 5,685 articles were excluded for reasons of focusing on things other than mechanisms, being unrelated to chemotherapy -induced peripheral neuropathy, or for treating with parallel therapy or pharmacopuncture. A 


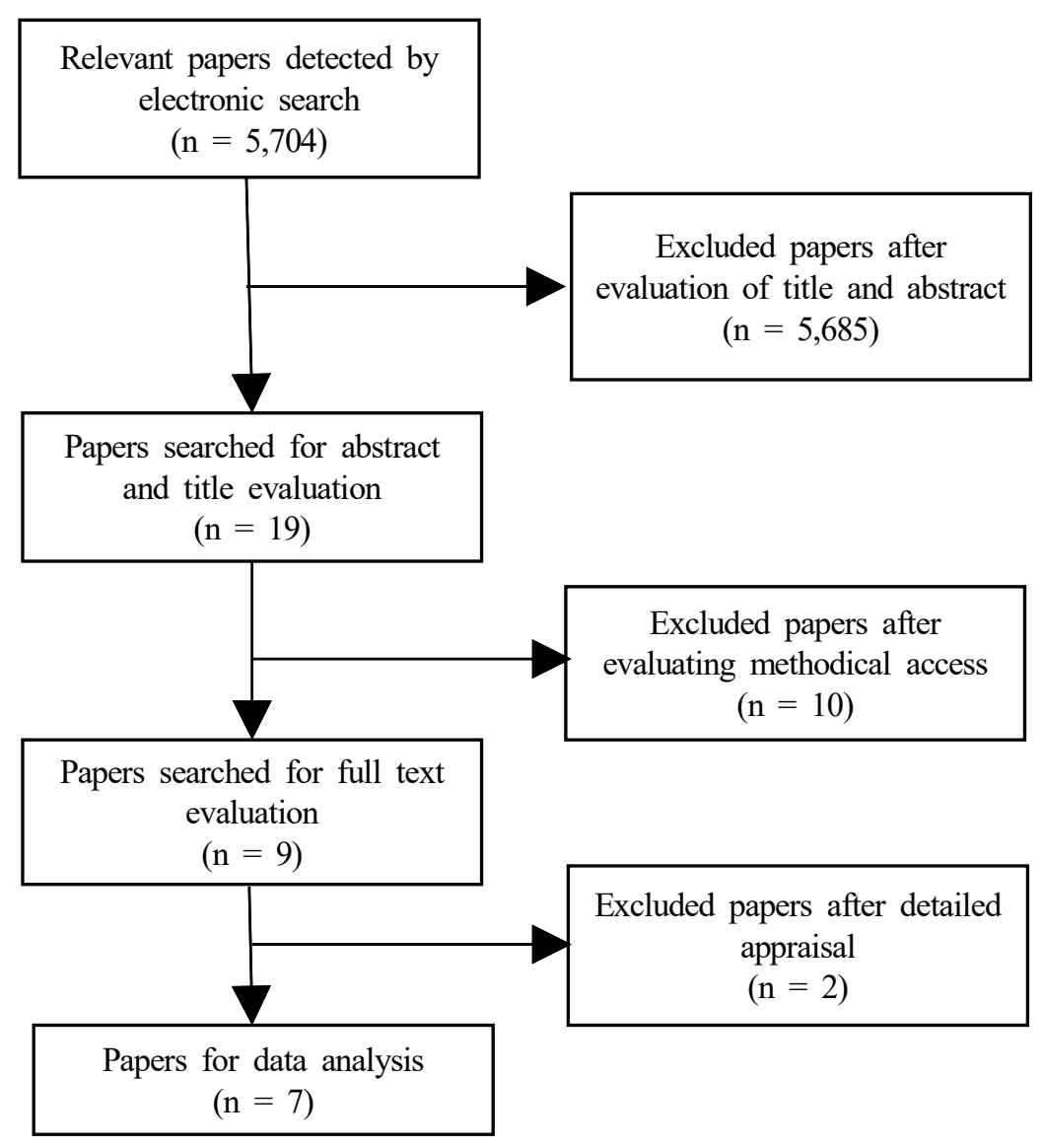

Fig. 1. Study selection for mechanisms of acupuncture for treating chemotherapy-induced peripheral neuropathy

total of 7 experimental articles were retrieved and mainly suggestive for this article (Figure 1).

\section{Results}

A total of 7 articles were retrieved for the mechanism of acupuncture for treating CIPN, 1 pilot study and 6 experimental studies were included in this study.

\section{Effect on Cytokines and Neurotransmitters}

In one pilot study 27 patients who were diagnosed multiple myeloma (MM) revealed bortezomib-induced peripheral neuropathy. ${ }^{7)}$ After the end of their treatment, a Functional Assessment of Cancer Therapy/Gynecologic Oncology Group -Neurotoxicity (FACT/GOG-Ntx) questionnaire, Total Neuropathy Score (TNSc), and the Neuropathy Pain Sale reduced, significantly indicating decreased pain and improved function $(\mathrm{p}<0.0001)$. However there were no valid changes not only in serum cytokines including macrophage inflammatory protein 1a (MIP-1a), vascular endothelial growth factor (VEGF), 
insulin-like growth factor-1 (IGF-1), and interleukin-6 (IL-6), IL-8, IL-10, IL-17a but also in serum neurotransmitters and neurohormones including nerve growth factor (NGF), brain -derived neurotrophic factor (BDNF), $\beta$-endorphin ( $\beta$-EP), and neurotrophin-3 (NT-3). However, there were limitations that the patients with MM may be affected by disease easily and there is not an established correlation between cerebrospinal fluid with peripheral tissue level and blood.

\section{Effect on $\beta$-Endorphin}

In an experimental study for experimenting on the effectiveness of EA on oxaliplatin-induced neuropathic pain, ${ }^{8)}$ low frequency $(2 \mathrm{~Hz}) \mathrm{EA}$ at ST36 alleviated cold allodynia more effectively than high frequency EA at ST36 in rats. They used opioid antagonist and $a$-adrenergic antagonist to investigate which receptor mediates to EA stimulation, that the opioid receptor antagonist completely blocked the EA effect $(p>0.05)$ where as a-adrenergic antagonist did not $(p<0.05)$. Also, 10 minutes after, at the end of EA stimulation, plasma $\beta$-endorphin levels increased significantly and decreased subsequently. In this study, it was found that low frequency EA at ST36 is mediated by the endogenous opioid resulting in alleviating effect on oxaliplatin-induced cold allodynia in rats.

In another study for experiments to examine the endogenous opioid mechanism for oxaliplatin -induced neuropathic in rats, ${ }^{9}$ acupoint ST36 was stimulated by EA. Both groups treated by EA for 20 minutes and 30 minutes showed markedly anti-allodynic effects. To confirm the mechanism of EA, plasma $\beta$-endorphin levels and spinal encephalin were measured. 10 minutes after the end of EA stimulation, plasma $\beta$ -endorphin levels increased significantly $(p<0.001)$, whereas no difference in spinal encephalin levels between the control group and EA groups was detected. As a result, the opioid mechanism can act on the effectiveness of EA to reduce cold allodynia-induced by oxaliplatin.

\section{Effect Through the $\mu, \delta$, and $\kappa$ Opioid Receptors}

In another experimental study for the effect of EA on paclitaxel(PTX)-induced peripheral neuropathy in rats, ${ }^{10)} 10 \mathrm{~Hz}$ EA relieved hyperalgesia and allodynia more than $100 \mathrm{~Hz}$ EA. Also, they investigated the how $10 \mathrm{~Hz}$ EA acts. EA-produced pain inhibition is blocked by a $\mu$ opioid receptor antagonist. EA + either the $\delta$ or $\kappa$ opioid receptor antagonist also did not decrease response, indicating that EA-produced inhibition of hyperalgesia and allodynia is blocked by $\delta$ or $\kappa$ opioid receptor antagonists (between $10 \mathrm{~Hz}$ EA $+\mu$ opioid receptor antagonist versus $10 \mathrm{~Hz}$ EA + vehicle, on 18 days, $\mu: \mathrm{p}<0.01$ at $4,8,15 \mathrm{~g}$ stimulation of PTX, $\mu: \mathrm{p}<0.01$ at $4,15 \mathrm{~g}$ stimulation of PTX and $\mathrm{p}<0.05$ at $6 \mathrm{~g}$ stimulation of PTX, $\kappa: \mathrm{p}<0.05$ at $4,8,15 \mathrm{~g}$ stimulation of PTX). In conclusion, $\mu, \delta$, and $\kappa$ opioid receptor subtypes can be involved in EA effects for relieving PTX-induced peripheral neuropathy.

\section{Effect Through $\mathrm{M}_{2}, \mathrm{M}_{3}, 5-\mathrm{HT}_{3}$ Receptors}

In another study to investigate the relieving effect of EA on oxaliplatin-induced neuropathic cold allodynia in rats, ${ }^{11)}$ EA stimulated at ST36, GV3, LI11. All of them showed an anti-allodynic effect. In addition, they confirmed that either muscarinic or nicotinic receptors are mediated in 
the effect of EA through injection of non -selective muscarinic acetylcholine(M) receptor antagonist (atropine) or non-selective nicotinic receptor antagonist (mecamylamine). Compared with Normal saline(NS) by measuring tail withdrawal latency(TWL), it is discovered that $\mathrm{M}$ receptors are mediated (NS: $\mathrm{p}<0.01$, atropine: $\mathrm{p}>0.05$, mecamylamine: $\mathrm{p}<0.01)$. Then, they injected NS, $M_{1}, M_{2}$, and $M_{3}$ receptor antagonists and DMSO, 5-hydroxytryptamie $(5-\mathrm{HT})_{1 \mathrm{~A}}, 5-\mathrm{HT}_{2 \mathrm{~A}}$, and $5-\mathrm{HT}_{3}$ receptor antagonists for finding which receptors are mediated in the effect of EA by measuring TWL. They confirmed that the spinal cholinergic including $\mathrm{M}_{2}$ and $\mathrm{M}_{3}$ can mediate the effect of EA (NS: $p<0.01, M_{1}: p<0.01, M_{3}: p>0.05$ ), and serotonergic $5-\mathrm{HT}_{3}$ receptors also mediate the effect of EA on analgesic action for oxaliplatin -induced neuropathic pain (DMSO: $\mathrm{p}<0.05,5-\mathrm{H}_{1 \mathrm{~A}}$ : $\left.\mathrm{p}<0.001,5-\mathrm{H}_{2 \mathrm{~A}}: \mathrm{p}<0.01,5-\mathrm{H}_{3 \mathrm{~A}}: \mathrm{p}<0.05\right)$.

\section{Effect Through a2- and \\ $\beta$-Adrenoceptors, Opioid Receptor}

In another study to affirm the effectiveness of EA and determining relation mechanism of EA and PTX-induced neuropathic pain, ${ }^{12)}$ in rats, EA stimulates acupoints ST36 bilaterally. For intrathecal pretreatment, opioid receptor antagonist naloxone (NAL), a2-adrenoceptor antagonist idazoxan (IDA), $\beta$-adrenoceptor antagonist propranolol (PRO), and a1-adrenoceptor antagonist prazosin (PRZ) were chosen. In the results, PTX-induced mechanical allodynia and thermal hyperalgesia were markedly alleviated by EA stimulation. In addition, it is confirmed that during EA stimulation at ST36, $\alpha 2-$ and $\beta$-adrenoceptors, the spinal opioid receptor can be included (at 120 minutes after EA stimulation, compared between PTX + EA group and PTX + adrenoceptor antagonist + EA, NAL: $\mathrm{p}<0.01$, IDA: $\mathrm{p}<0.05$, PRO: $\mathrm{p}<0.01)$.

\section{Effect Through 5-HT $1 \mathrm{~A}$ Receptors and p-CaMKII}

The last study to demonstrate the effectiveness of EA on PTX induced neuropathic pain in rats. ${ }^{13)}$ Bilateral acupoints GB30 were stimulated by EA. As for sham control, EA needles were administered into GB30 without stimulation. And to investigate the roles of $5-\mathrm{HT}_{1 \mathrm{~A}}$ receptors, $5-\mathrm{HT}_{1 \mathrm{~A}}$ receptor antagonist (WAY-100635) was applied resulting in indicating that the mechanism of EA includes $5-\mathrm{HT}_{1 \mathrm{~A}}$ receptor activation (on 18 days, comparing between EA+vehicle and EA+WAY-100635, $\mathrm{p}<0.01$ at 4, $15 \mathrm{~g}$ stimulation of PTX, $<<0.05$ at $8 \mathrm{~g}$ stimulation of PTX). Moreover, they confirmed that EA decreased p-calmodulin-dependent protein kinase(CaMKII) levels compared with sham in the spinal cord $(p<0.01)$. Besides, CaMKII were affected through the activation of $5-\mathrm{HT}_{1 \mathrm{~A}}$ receptors in the spinal cord. In conclusion, spinal $5-\mathrm{HT}_{1 \mathrm{~A}}$ receptors is activated to inhibit $\mathrm{p}$-CAMKII to alleviate PTX-induced pain by EA.

Through these studies, not only did electroacupunture alleviate both allodynia and hyperalgesia caused by chemotherapy but we could also find that 5-HT, especially activating $5-\mathrm{HT}_{1 \mathrm{~A}}$ receptor and opioids, especially $\beta$ -endorphin, could be related to inhibiting pain induced by chemotherapy. Furthermore, it is not clear that adrenaline mediates the EA-pain control system by activating adrenoceptors. Also other neurotransmitters such as muscarine and encephalin require further research (Table 1.) (Table 2.). 


\section{Discussion}

Most of the drug induced neuropathy affects nerve fibers and neuronal bodies, especially to dorsal root ganglia. Most especially, antineoplastic agents cause a major clinical problem. In fact, the peripheral neuropathy frequently represents as a dose limiting side effect. And its onset may affect the quality of life of cancer patients and cause discomfort. Moreover, in some cases of CIPN it's only partly reversible while symptoms may resolve. ${ }^{14)}$ In other cases it isn't reversible at all. CIPN can be severely painful, disabling, and cause functional abilities eventually decreasing the quality of life.

The representative drugs which cause chemotherapy -induced peripheral neuropathy (CIPN) include platinum drugs, taxanes, epothilones, vinca

Table 1. References for the Mechanisms of Chemotherapy-Induced Peripheral Neuropathy

\begin{tabular}{|c|c|c|c|}
\hline Authors & Subject & Journal & Year Published \\
\hline Bao, T. et al. & $\begin{array}{c}\text { "A Pilot Study of Acupuncture in Treating } \\
\text { Bortezomib-Induced Peripheral Neuropathy in Patients } \\
\text { with Multiple Myeloma" }\end{array}$ & Integrative Cancer Therapies & 2014 \\
\hline Moon, HJ. et al. & $\begin{array}{l}\text { "Effects of Electroacupuncture on Oxaliplatin-Induced } \\
\text { Neuropathic Cold Hypersensitivity in Rats" }\end{array}$ & $\begin{array}{l}\text { The Journal of Physiological } \\
\text { Sciences }\end{array}$ & 2014 \\
\hline Lee, DI. & $\begin{array}{l}\text { "Anti-Allodynic Effect of Electoracupuncutre in } \\
\text { Oxaliplatin-Induced Neuropathic Rats: The } \\
\text { Endogenous Opioid Mechanism" }\end{array}$ & $\begin{array}{c}\text { Doctoral Dissertation, Kyung } \\
\text { Hee University }\end{array}$ & 2014 \\
\hline Meng, X. et al. & $\begin{array}{l}\text { "The Effects of Opioid Receptor Antagonists on } \\
\text { Electroacupuncture-Produced } \\
\text { Anti-Allodynia/Hyperalgesia in Rats with } \\
\text { Paclitaxel-Evoked Peripheral Neuropathy" }\end{array}$ & Brain Research & 2011 \\
\hline Lee, JH. et al. & $\begin{array}{l}\text { "Involvement of Spinal Muscarinic and Serotonergic } \\
\text { Receptors in the Anti-Allodynic Effect of } \\
\text { Electroacupuncture in Rats with Oxaliplatin-Induced } \\
\text { Neuropathic Pain" }\end{array}$ & $\begin{array}{c}\text { Korean Journal of Physiology \& } \\
\text { Pharmacology }\end{array}$ & 2016 \\
\hline $\begin{array}{l}\text { Choi, JW., Kang, SY., } \\
\text { Choi, JG. et al. }\end{array}$ & $\begin{array}{l}\text { "Analgesic Effect of Electroacupuncture on } \\
\text { Paclitaxel-Induced Neuropathic Pain via Spinal } \\
\text { Opioidergic and Adrenergic Mechanisms in Mice" }\end{array}$ & $\begin{array}{l}\text { The American Journal of } \\
\text { Chinese Medicine }\end{array}$ & 2015 \\
\hline $\begin{array}{l}\text { Zhang, Y., Li A., Xin, J. } \\
\text { et al. }\end{array}$ & $\begin{array}{l}\text { "Electroacupuncture Alleviates } \\
\text { Chemotherapy-Induced Pain Through Inhibiting } \\
\text { Phosphorylation of Spinal CaMKII in Rats" }\end{array}$ & European Journal of Pain & 2017 \\
\hline
\end{tabular}

CaMKII, Calmodulin-dependent protein kinase II.

Table 2. Mediated Substance or Receptors for EA Stimulation

\begin{tabular}{ccc}
\hline Authors & Mediated Substance or Receptor & Chemotherapy Drugs \\
\hline Bao, T., et al. & No change in cytokines and neurotransmitters & Bortezomib \\
Moon, HJ., et al. & $\beta$-endorphin & Oxaliplatin \\
Lee, DI.. & $\beta$-endorphin & Oxaliplatin \\
Lee, JH., et al. & $\mathrm{M}_{2}, \mathrm{M}_{3}$, 5-HT and 5-HT receptor & Oxaliplatin \\
Choi, JW., Kang, SY., Choi, JG. et al. & $a$ 2- and $\beta$-adrenoceptors, spinal opioid receptor & Paclitaxel \\
Meng, X., et al & $\mu, \delta$ and $\kappa$ opioid receptor & Paclitaxel \\
Zhang, Y., Li, A., Xin, J., et al. & $5-\mathrm{HT}_{1 \mathrm{~A}}$ receptors and & Paclitaxel \\
\hline
\end{tabular}

A, Electoroacupunture; 5-HT, 5-hydroxytryptamine; CaMKII, Calmodulin-dependent protein kinase II. 
alkaloids, and bortezomib. ${ }^{15)} \mathrm{A}$ number of factors are influential on CIPN including a patient's age, dose intensity, cumulative dose, co-administration, and therapy duration. ${ }^{16)}$

Compared with other neuropathic pain, CIPN has similar symptoms to diabetic neuropathy as the feeling of glove and stocking distribution, pain, and paresthesia, as usual clinical symptoms begin with paresthesia and dysesthesia, which occurs in fingers and toes. These symptoms spread to both extremities in characteristic 'glove and stocking' distribution. ${ }^{17)}$

However the mechanism of neurotoxicity of the chemotherapy drugs is yet unclear. It is estimated and known that CIPN is caused through multiple mechanisms as a disruption of microtubule mediated transport, axonal degeneration, oxidative stresses, and mitochondrial damage, blocking ion channels ${ }^{18)}$

Acupuncture treatment has been raised as a treatment for CIPN according to some articles. Lots of cases report that the acupuncture treatment is effective to decrease symptoms of CIPN. In one prospective case series, using acupuncture and selecting CV6, ST36, LI11, EX-LE10, and EX-UE9 acupoints, it can decrease the pain score with no side effects. ${ }^{19)}$ And in another retrospective case series, the 18 patients who were diagnosed with CIPN, 14 patients had decreased symptoms of neuropathy after 6 weeks of treatment using SP6, ST36, and LV3 acupoints. $^{20)}$

As a pain control system using acupuncture, a descending inhibitory pathway system has been generally suggested. This system is called the analgesia system. When the muscle sensory afferents is disturbed, the dorsal horn of the spinal cord acts as important role. The neurons which originate from the periaqueductal gray, the main control area modulating the descending system in mesencephalon, send their signals to the nuclei of raphe. And the reticular activating system located in the rostral medulla level is where the descending anti-nociceptive track originates. Excitatory amino acids activate the cells in this area. Acupuncture could affect pain perception by modulating the activity of the subcortical and brainstem sites through the descending pathway and causing them to secret endogen opioids. These stimuli go through the dorsolateral column of the medulla spinal. These stimuli could then promote neurotransmitters such as encephalin from local neurons in the medulla spinalis and serotonin from nerve fibers originated in the raphe magnus. ${ }^{21)}$

The studies included in this review: 1 pilot study and 6 experimental studies. In one pilot study, ${ }^{7)} 27$ patients were targeted who were diagnosed multiple myeloma and had revealed bortezomib-induced peripheral neuropathy (grade $\geq 2$ ). They were treated by acupuncture completely. Acupoints included bilateral ear points (sin mun and 2 electro-dermal signal detected acupoints), bilateral body points (LI4, TE5, LI11, ST40), and EX-LE8. For these acupoints, the disposable sterilized acupuncture needles (Seirin Co, Shizuoka, Japan) were inserted into the skin. The Neuropathy Pain Scale (NPS), the Clinical Total Neuropathy Score (TNSc), and the Functional Assessment of Cancer Therapy/Gynecologic Oncology Group-Neurotoxicity (FACT/GOG-Ntx) questionnaire were used for responses. Due to TNSc some data was deemed invalid, and it was excluded from this study. Among the 25 patients who completed acupuncture at least 4 times, $56 \%(\mathrm{n}=14)$ reported improvement in daily 
functions, $40 \%(\mathrm{n}=10)$ reported more than a $50 \%$ decrease in NPS, and $28 \%(n=7)$ reported more than a $50 \%$ reduction in FACT/GOG-Ntx score. They used cytokines which were MIP-1a, VEGF, IGF-1, IL-6, IL-8, IL-10, and IL-17a. In addition, they contained neurotrophic factors which were NGF, BDNF, $\beta-E P$, and NT-3. There were no significant changes in cytokines or neutrophic factors measured in the 10 weeks of treatments. They concluded that the correlation between cerebrospinal fluid with peripheral tissue and blood in spite of neutrophic factors found in the serum could not be established.

In another experimental study for experimenting with effectiveness of EA on oxaliplatin-induced neuropathic pain, ${ }^{8)}$ rat model was targeted. To confirm hypersensitivity in a rat model, cold allodynia were evaluated by immersing the tail into $4{ }^{\circ} \mathrm{C}$ cold water and then measured by withdrawal latency. It was injected 3 days after oxaliplatin administration (6 $\mathrm{mg} / \mathrm{kg})$ had been done. For the acupoint, ST36 were selected, and sham was selected at a non-acupoint. EA (2/100 $\mathrm{Hz}, 0.2-0.3 \mathrm{~mA}, 0.3-\mathrm{ms}$ pulse duration) stimulated these points for 20 minutes. In the results, it was more effective in relieving cold allodynia in the case of low-frequency $(2 \mathrm{~Hz})$ than low-frequency EA at non-acupoints or high-frequency EA at ST36. Although phentolamine $(2 \mathrm{mg} / \mathrm{kg})$ could not block EA-induced anti-allodynia $(p<0.05)$, naloxone $(2 \mathrm{mg} / \mathrm{kg})$ did block $(\mathrm{p}>0.05)$. Moreover, at 10 minutes after the end of EA stimulation, $\beta$ -endorphin levels significantly increased and subsequently decreased. Morphine hydrochloride showed a stronger, but similarly lasting to EA-induced anti-allodynic effect. However, more prolonged analgesic action was shown on EA stimulation (30 min EA) than morphine.
In another study for experimental examining of the endogenous opioid mechanism for oxaliplatin-induced neuropathic in rats, ${ }^{9)}$ acupoint ST36 was stimulated by EA. For EA stimulation (2 Hz, 0.5 ms, 0.2-0.3 mA) was conducted during $20(n=5)$ or $30(n=6)$ minutes in different groups. Morphine hydrochloride was injected intraperitoneally $2 \mathrm{mg} / \mathrm{kg}$ at the same time as EA stimulation. Compared with the control group, both the 20-minute and 30-minute EA groups showed significant anti-allodynic effects. That effect gradually decreased 90 minutes after the end of EA stimulation in the 20-minute group, and up to 180 minutes in the 30 -minute group. Plasma $\beta$-endorphin levels $(n=4)$ rose 10 minutes after the end of EA stimulation $(\mathrm{P}<0.001)$. However spinal encephalin levels were not different between the control group and EA groups, so it is concluded that the EA affects the cold allodynia model induced by oxaliplatin through an opioid mechanism.

As another experimental study, ${ }^{10}$ researchers evaluated the effect of EA on PTX-induced peripheral neuropathy. They described PTX -induced responses using $15 \mathrm{~g}$ of pressure as hyperalgesia and $<15 \mathrm{~g}$ as allodynia. EA were stimulated throughout a 30-minute period. A 10 $\mathrm{Hz}$ EA treatment dramatically $(\mathrm{p}<0.05)$ decreased the response frequency at a stimulation of 4,8 , and $15 \mathrm{~g}$ pressure compared with sham EA. A $100 \mathrm{~Hz}$ EA treatment decreased response frequency at $15 \mathrm{~g}$ only. It could be indicatable that $10 \mathrm{~Hz}$ EA potentially inhibited the hyperalgesia and allodynia more than $100 \mathrm{~Hz}$ EA. They also investigated how the $10 \mathrm{~Hz}$ EA acts. Three opioid receptor antagonists, CTOP (D-Phe-Cys-Tyr-D-Trp-Orn-Thr-Pen-ThrNH2) for $\mu$, NTI (naltrindole hydrochloride) for $\delta$, or BNI 
(nor-Binaltorphimine dihydrochloride) for $\kappa$ were selected and administered into the spinal cord. Compared with sham EA+vehicle, EA+CTOP did not significantly decrease the response frequency indicating that EA-produced pain inhibition is blocked by this antagonist. EA + either the $\delta$ or $\kappa$ opioid receptor antagonist also did not significantly decrease the response frequency compared with sham $\mathrm{EA}+$ vehicle. It could also be indicating that the EA-produced inhibition of hyperalgesia and allodynia is blocked by $\delta$ or $\kappa$ opioid receptor antagonists. So, in this study, all three opioid receptor subtypes could be involved in EA effects for treating PTX-induced peripheral neuropathy.

In another study focusing on the spinal cholinergic and serotonergic analgesic system which could control oxaliplatin-induced neuropathic cold allodynia by using $\mathrm{EA}^{11)}$ was led to a rat experiment. Oxaliplatin was injected to induce the cold allodynia. It was evaluated by soaking the rat's tail into cold water and then the withdrawal latency was measured. Acupoints were selected at ST36, GV3, or LI11. EA (2 Hz, $0.3-\mathrm{ms}$ pulse duration, $0.2 \sim 0.3 \mathrm{~mA}$ ) was stimulateed at these acupoints. Three different agents were administered with EA to investigate the cholinergic receptors in the effect of EA. These were NS, atropine (1 $\mathrm{mg} / \mathrm{kg})$, and mecamylamine (2 $\mathrm{mg} / \mathrm{kg})$. In addition, to confirm the analgesic effect of EA from which $M$ receptor subtypes mediate, pirenzepine $\left(\mathrm{M}_{1}\right.$ receptor antagonist, $10 \mu \mathrm{g})$, methoctramine $\left(\mathrm{M}_{2}\right.$ receptor antagonist, $10 \mu \mathrm{g})$, and 4-DAMP $\left(\mathrm{M}_{3}\right.$ receptor antagonist, $10 \mu \mathrm{g}$ ) were administered intrathecally before 20 minutes of treatment with EA. Moreover, NAN-190 $\quad\left(5-\mathrm{HT}_{1 \mathrm{~A}}\right.$ receptor antagonist, $15 \mu \mathrm{g})$, ketanserin $\left(5-\mathrm{HT}_{2 \mathrm{~A}}\right.$ receptor antagonist, $30 \mu \mathrm{g})$, and MDL-72222 $\left(5-\mathrm{HT}_{3}\right.$ receptor antagonist, $12 \mu \mathrm{g}$ ) were injected before 20 minutes of treatment with EA for observing the effect of serotonergic receptor subtypes in the analgesic mechanism of EA. As a result, all acupoints stimulated with EA showed the potency of EA for decrease allodynia. Most especially, GV3 and ST groups showed more significant changes in withdrawal latency than LI11 (LI11 : $\mathrm{p}<0.05$, GV3: $\mathrm{p}<0.01$, ST36: $\mathrm{p}<0.001)$. Also, the analgesic effect of EA in the ST36 and GV3 groups lasted until one hour after the stimulation. There was a markedly increase in tail withdrawal latency (TWL) $(\mathrm{p}<0.01)$ in the NS group indicating the analgesic effect of EA on cold allodynia in rats. After intraperitoneal administration, neither atropine nor mecamylamine changed TWL, which indicates that $M$ receptors without nicotinic acetylcholine receptors could be affected to relieve the effect of EA at oxaliplatin-induced cold allodynia. The relieving effects of on cold allodynia were not blocked by the injection of NS or pirenzepine $(p<0.01)$, However, methoctramine and 4-DAMP did block the analgesic effect of EA $(p>0.05)$. These results indicate that acetylcholine receptor subtypes in spinal $\mathrm{M}_{2}$ and $\mathrm{M}_{3}$ without $\mathrm{M}_{1}$ are affected by the analgesic effect of EA. There was a significant increase in TWL in the DMSO group $(\mathrm{p}<0.05)$ indicating the analgesic effect of EA. MDL-72222 markedly blocked the analgesic effect of EA whereas NAN-190 and ketanserin did not. So, $5-\mathrm{HT}_{3}$ receptors could be involved in the analgesic effect of EA for controlling oxaliplatin-induced cold allodynia in rats.

In another study for determining the antinociceptive effect and relation with neuronal mechanism of EA on PTX-induced neuropathic 
pain ${ }^{12)}$ to induce neuropathic pain, PTX (4 mg/ $\left.\mathrm{kg}\right)$ was injected once a day for 5 consecutive days in rats. EA stimulates $(2 \mathrm{~mA}, 2 \mathrm{~Hz}, 30 \mathrm{~min})$ happened every 2 days and acupoints were selected on ST36 bilaterally. Four intrathecal pretreatments were done which were the following: naloxone (opioid receptor antagonist), idazoxan (a2-adrenoceptor antagonist), propranolol ( $\beta$-adrenoceptor antagonist), and prazosin ( $\alpha 1$-adrenoceptor antagonist). In a set of experiments, in the case of intrathecal pretreatment with naloxone, idazoxan or propranolol reduced the anitinociceptive single EA stimulation effect 8 days after PTX treatment, whereas prazosin did not. The EA stimulation markedly decreased the PTX-induced mechanical allodynia and thermal hyperalgesia. In addition, the PTX-enhanced phosphorylation of the N-Methyl-D-aspartic acid (NMDA) receptor N-methyl D-aspartate receptor subtype 2B (NR2B) subunit in the spinal dorsal horn is reduced by EA stimulation $(\mathrm{p}<0.05)$. And it is confirmed that intrathecal pretreatment of naloxone, idazoxan, or propranolol blocked the analgesic effect of EA. As a result, the mediation of spinal opioid receptors, $\alpha 2-$ and $\beta$ -adrenoceptors, could be included to reduce PTX-induced neuropathy by using EA stimulation at ST36.

In another study for demonstrating the effectiveness of electroacupuncture on PTX -induced neuropathic pain in a rat model, ${ }^{13)}$ they injected PTX (2 $\mathrm{mg} / \mathrm{kg}$ ) or vehicle on days $0-6$. The selected acupoint was GB30 and it was stimulated bilaterally at $10 \mathrm{~Hz}, 2 \mathrm{~mA}, 0.4 \mathrm{~ms}$ pulse throughout 30 minutes by EA. As for sham control, EA needles were administered into GB30 without stimulation. And to investigate the roles of $5-\mathrm{HT}_{1 \mathrm{~A}}$ receptors on the effect of $\mathrm{EA}$ on
PTX-induced pain, rats were injected with PTX and divided into four groups which were the following: WAY-100635 (5- $\mathrm{HT}_{1 \mathrm{~A}}$ receptor antagonist)+ EA, WAY-100635+sham, saline+EA, and saline+sham. The EA significantly alleviated PTX-induced allodynia and hyperalgesia. And WAY-100635 in EA-treated rats increased the mechanical response frequencies compared to the vehicle indicating that WAY-100635 blocked EA inhibition of allodynia and hyperalgesia. These results suggest that the process of EA-produced relief of chemotherapy induced pain includes $5-\mathrm{HT}_{1 \mathrm{~A}}$ receptor activation. Moreover, to investigate the EA effect on p-CaMKII, the rats were divided into four groups and the spinal cord was removed after finishing the behavioral test in the $5-\mathrm{HT}_{\mathrm{A}}$ receptor experiment. Those PTX-injected rats with saline and sham EA groups showed markedly higher levels of p- CaMKII. And the EA treatment decreased p-CaMKII levels compared with sham. Also, the pretreatment of intrathecal 5- $\mathrm{HT}_{1 \mathrm{~A}}$ receptor antagonist increased the p-CAMKII levels in EA-treated rats compared with the vehicle pretreatment indicating that EA may inhibit phosphorylation of CaMKII through activation of $5-\mathrm{HT}_{1 \mathrm{~A}}$ receptors in the spinal cord. In conclusion, EA activates spinal $5-\mathrm{HT}_{1 \mathrm{~A}}$ receptors for inhibiting p-CAMKII to alleviate PTX-induced pain.

Therefore, it is suggestable that the mechanism of EA is based on the descending inhibitory pathway especially medicated by $5-\mathrm{HT}$ and $\beta$ -endorphin which were confirmed from these studies.

\section{Conclusion}

The data from the present studies show that low-frequency EA are effective for CIPN. Also, 
they mediated the endogenous opioid and 5-HT in the spinal cord and serums. Thus, we suggest that to inhibit CIPN, EA treatment can be used to control pain through the descending inhibitory pathway by being mediated by the 5-HT and endogenous opioids including $\beta$-endorphin and encephalin in the spinal cord and serums. Addtional experiments should be carried out in the future.

\section{Acknowledgements}

We thank colleagues in Public health center of Jeungpyeong for constructive advices and researchers in Daejoen Korean Medicine Hospital for medical suggestions.

\section{References}

1. Majithia N, Loprinzi CL, Smith TH. New Practical Approaches to Chemotherapy-Induced Neuropathic Pain: Prevention, Assessment, and Treatment, Oncology. 2016; 30(11):1020 $-1029$.

2. Kim PY, Johnson, Cerrie E. Chemotherapy -induced peripheral neuropathy: a review of recent findings, Current Opinion in Anesthesiology. 2017; 30(5): 570-576.

3. Sherry W, Debra B, Lisa $\mathrm{K}$ et al. Chemotherapy-induced peripheral neuropathy: Prevention and treatment strategies, European Journal of Cancer. 2008; 44(11):1507-1515.

4. Deng GE, Frenkel M, Cohen $\mathrm{L}$ et al. Evidence-based clinical practice guidelines for integrative oncology: complementary therapies and botanicals, The Journal of Society of Integrative Oncology. 2009; 7(3): 85-120.
5. Rossi E, Vita A, Baccetti $\mathrm{S}$ et al. Complementary and alternative medicine for cancer patients: results of the EPAAC survey on integrative oncology centres in Europe, Supportive Care in Cancer. 2015; 23(6): 1795-1806.

6. Zhang C, Ma T, Yan Y. Clinical effects of acupuncture for diabetic peripheral neuropathy, Journal of Traditional Chinese Medicine. 2010; 30(1): 13-14.

7. Bao T, Goloubeva O, Pelser C. et al. A Pilot Study of Acupuncture in Treating Bortezomib-Induced Peripheral Neuropathy in Patients With Multiple Myeloma, Integrative Cancer Therapies. 2014; 13(5): 396-404.

8. Moon HJ, Lim BS, Lee DI et al. Effects of electroacupuncture on oxaliplatin-induced neuropathic cold hypersensitivity in rats, The journal of Physiological Sciences. 2014; 64(2): 151-156.

9. Lee DI. Anti-allodynic Effect of Electoracupuncutre in Oxaliplain-induced Neuropathic Rats: The Endogenous Opioid Mechanism, Doctoral Dissertation, Kyung Hee University, 2014.

10. Meng X, Zhang Yu, Li A et al. The effects of opioid receptor antagonists on electroacupuncture -produced anti-allodynia/hyperalgesia in rats with paclitaxel-evoked peripheral neuropathy, Brain research. 2011; 1414(1): 58-65.

11. Lee JH, Go DH, Kim WJ et al. Involvement of spinal muscarinic and serotonergic receptors in the anti-allodynic effect of electroacupuncture in rats with oxaliplatin-induced neuropathic pain. Korean Journal of Physiology \& Pharmacology, 2016; 20(4): 407-414.

12. Choi JW, Kang SY, Choi JG et al. Analgesic Effect of Electroacupuncture on 
Paclitaxel-induced Neuropathic Pain via Spinal Opioidergic and Adrenergic Mechanisms in Mice, The American Journal of Chinese Medicine. 2015; 43(1): 57-70.

13. Zhang Y, Li A, Xin J et al. Electroacupuncture alleviates chemotherapy-induced pain through inhibiting phosphorylation of spinal CaMKII in rats, European Journal of Pain. 2017; 21(9): 1-12.

14. Quasthoff S, Hartung HP. Chemotherapy -induced peripheral neuropathy, Journal of neurology. 2002; 249(1): 9-17.

15. Argyriou AA, Bruna J, Marmiroli P. Chemotherapy-induced peripheral neurotoxicity (CIPN): An update, Critical Reviews in Oncology/Hematology. 2012; 82(1): 51-77.

16. Windebank AJ, Grisold W. Chemotherapy -induced neuropathy, Journal of the peripheral Nerve society. 2008; 13(1): 27-46.

17. LoMonaco M, Milone M, Batocchi AP, et al. Cisplatin neuropathy: clinical course and neurophysiological findings, Journal of Neurology. 1992; 239(4): 199-204.

18. Boyette-Davis JA, Walters ET, Dougherty PM. Discovering cytokines as targets for chemotherapy-induced painful peripheral neuropathy, Cytokine. 2012; 59(1): 3-9.

19. Wong R, Sagar S. Acupuncture treatment for chemotherapy-induced peripheral neuropathy-a case series, Acupuncture in Medicine. 2006; 24(2): 87-91.

20. Donald GK, Tobin I, Stringer J. Evaluation of acupuncture in the management of chemotherapy-induced peripheral neuropathy, Acupuncture in Medicine. 2011; 29(3): 230-233.

21. Audette JK, Ryan AH. The role of acupuncture in pain management, Physical Medicine \& Rehabilitation Clinics. 2004; 15(4): 749-772.

\section{ORCID}

Hyunsik Seo https://orcid.org/0000-0002-7264-1638

Jung-hyo Cho https://orcid.org/0000-0002-1094-0165

Chang-gue Son https://orcid.org/0000-0003-4876-0167

Nam-Hun Lee https://orcid.org/0000-0002-5039-2423 